\title{
Avaliação de Clones de Capim-Elefante (Pennisetum purpureum Schum.) e de um Híbrido com o Milheto (Pennisetum glaucum (L.) R. Br.) Submetidos a Estresse Hídrico. 1. Parâmetros Morfológicos ${ }^{1}$
}

\author{
Glesser Porto Barreto², Mário de Andrade Lira ${ }^{3}$, Mércia Virgínia Ferreira dos Santos ${ }^{4}$, \\ José Carlos Batista Dubeux Júnior ${ }^{4}$
}

\begin{abstract}
RESUMO - Este trabalho foi conduzido com o objetivo de avaliar características morfológicas de três cultivares de capim-elefante (Cameroon, Roxo de Botucatu e Mott) e de um híbrido de capim-elefante com o milheto (Híbrido HV-241), submetidos a dois regimes de umidade (com e sem estresse hídrico). Utilizou-se o delineamento em blocos casualizados com parcelas subdivididas e três repetições. Na parcela principal, estudou-se o efeito dos regimes de umidade e nas subparcelas, os diferentes clones. As plantas submetidas a estresse hídrico apresentaram menor altura e nenhuma delas apresentou internódios acima da altura de corte $(10 \mathrm{~cm})$. A redução na altura das plantas, como consequência do estresse, foi de 42,7\% (Cameroon), 35,05\% (Roxo de Botucatu), 28,54\% (híbrido HV-241) e 27,43\% (Mott). O comprimento da lâmina foliar foi reduzido de $69,9 \mathrm{~cm}$, nas plantas irrigadas, para 50,0 cm nas submetidas a estresse, enquanto a largura da lâmina foliar das plantas sob estresse correspondeu a menos da metade das plantas irrigadas. O estresse hídrico não influenciou o perfilhamento dos cultivares, mas, no híbrido HV-241, reduziu o número de perfilhos axilares/planta e o número total de perfilhos/ planta. Em ambos os regimes de umidade, o híbrido HV-241 foi o material que apresentou o maior perfilhamento. Observou-se que, com exceção do perfilhamento nos cultivares, o estresse hídrico promoveu redução nos demais parâmetros morfológicos estudados.
\end{abstract}

Palavras-chave: altura da planta, perfilhamento, tamanho da folha

\section{Evaluation of Elephant Grass Clones (Pennisetum purpureum Schum.) and Elephant Grass x Pearl Millet (Pennisetum glaucum (L.) R. Br.) Hybrid Submitted to Water Stress. 1. Morphological Parameters}

\begin{abstract}
This trial was carried out to evaluate the morphological characteristics of three Elephant grass clones (Cameroon, Roxo de Botucatu and Mott) and an Elephant grass with pearl millet hybrid (HV-241) submitted to two humidity regimes (with and without water stress). A randomized block design with split plots and three replicates was used. In the main plot, the effect of the humidity regimes was studied, and in the split plot, the different clones. Plants submitted to water stress showed lower height, and none of them showed internodes above cutting height $(10 \mathrm{~cm})$. As a result of water stress, the plant height reductions were $42.7 \%$ (Cameroon), 35.05\% (Roxo de Botucatu), 28.54\% (hybrid HV-241) and 27.43\% (Mott). Leaf blade length was reduced from $69.9 \mathrm{~cm}$, in the wet treatment, to $50.0 \mathrm{~cm}$ in those submitted to water stress, whereas leaf blade width average of water stress plants was less than a half of that in wet treatment plants. Water stress did not disturb tillering of cultivars but, in HV-241 hybrid, it reduced both the number of lateral tillers/ plant and total number of tillers/plant. In both humidity regimes, hybrid HV-241 was the clone, which showed the largest tillering. It was observed that, except for tillering of cultivars, water stress promoted a reduction on the other morphologic studied parameters.
\end{abstract}

Key Words: leaf size, plant height, tillering

\section{Introdução}

As plantas raramente crescem em ambientes ideais; muitas vezes, experimentam flutuações ambientais e estresses que modificam a sua morfologia e a taxa de desenvolvimento, limitam a produção e alteram a qualidade (BUXTON e FALES, 1994). A ocorrência de estresses hídricos moderados é comum.
Todavia, níveis mais severos de estresse hídrico são freqüentemente prejudiciais e detrimentais para o desenvolvimento normal das plantas (MEIDNER e SHERIFF, 1976). Na maior parte das áreas tropicais, o déficit hídrico é o fator mais importante na determinação do crescimento e da produtividade das forrageiras (SUÁREZ et al., 1986).

De maneira geral, todos os aspectos ligados ao

\footnotetext{
1 Parte da dissertação de mestrado do primeiro autor, financiada pela CAPES, FACEPE, UFRPE.

2 Engo-Agro, M.Sc. em Produção Animal (UFRPE), R. Dep Balduíno M. de Carvalho, 155 - apto. 603, Bessa, João Pessoa - PB, CEP 58.035-390. E.mail: glesser@bol.com.br

3 Pesquisador do IPA, bolsista do CNPq. E-mail: mlira@hotlink.com.br

4 Prof. do Departamento de Zootecnia da UFRPE. E.mail: rmsantos@elogica.com.br; dubeux@netpc.com.br
} 
crescimento da planta são afetados pelo estresse hídrico (KRAMER, 1983), sendo freqüentemente citadas, na literatura, a diminuição da expansão foliar (LEA et al., 1992), a aceleração na taxa de senescência foliar (BEGG, 1980), a inibição do perfilhamento e ramificações e a aceleração da morte dos perfilhos estabelecidos (BUXTON e FALES, 1994), bem como atraso no crescimento e no desenvolvimento da planta HSIAO e ACEVEDO (1975).

O conhecimento do comportamento da planta forrageira em condições de estresse hídrico pode, portanto, ser de grande importância prática para auxiliar no entendimento dos efeitos do período seco na produção de forragem, possibilitando, assim, o uso de práticas de manejo que tornem possível a melhor utilização do pasto durante esse período (DIAS FILHO et al., 1989).

Este trabalho foi conduzido com o objetivo de avaliar características morfológicas de três cultivares de capim-elefante e de um híbrido de capim-elefante com o milheto, submetidos a dois regimes de umidade.

\section{Material e Métodos}

O experimento foi conduzido em casa de vegetação, no Departamento de Zootecnia da Universidade Federal Rural de Pernambuco, em Recife - PE.

Como germoplasma, foram utilizados três cultivares de capim-elefante (Pennisetum purpureum Schum.): Cameroon, Roxo de Botucatu e Mott; e um híbrido interespecífico de capim-elefante com o milheto (Pennisetum glaucum (L.) R. Br.): Híbrido HV-241, cujos progenitores são o capim-elefante B e a linhagem macho estéril 23A do milheto.

Para o plantio, foram utilizadas mudas obtidas a partir de colmos fracionados em estacas contendo apenas uma gema cada, cultivadas em copos descartáveis de $500 \mathrm{~mL}$ de capacidade, contendo vermiculita expandida, e irrigadas diariamente até a data do transplantio. As plantas foram cultivadas em tanques de fibrocimento com 250 litros de capacidade. Cada tanque foi dividido em quatro partes, sendo que, na linha central de cada quadrante, foram plantadas cinco mudas do mesmo clone, de forma que cada tanque continha quatro clones diferentes, posicionados aleatoriamente.

Após um período de estabelecimento de 47 dias, realizou-se um corte de uniformização e, em seguida, a diferenciação dos tratamentos, quando a metade dos tanques teve a irrigação suspensa até a colheita do material, 36 dias depois. Por ocasião do transplantio e de cada corte (uniformização e colheita), foi realizada a aferição do teor de umidade do solo de cada tanque para a capacidade de campo, com base na determinação da umidade pelo método gravimétrico. As irrigações foram realizadas semanalmente, durante o $1^{\mathrm{o}}$ crescimento, e duas vezes por semana durante o $2^{\mathrm{o}}$ crescimento, com o objetivo de manter o solo próximo à capacidade de campo.

Por ocasião da colheita, realizada a $10 \mathrm{~cm}$ do solo, foram avaliados os seguintes parâmetros: altura da planta, $\mathrm{n}$ o de internódios por perfilho, comprimento e largura da lâmina foliar e número de perfilhos basilares, axilares e total por planta.

O delineamento experimental utilizado foi blocos ao acaso com parcelas subdivididas, com três repetições. Os blocos foram formados por conjuntos de dois tanques, cada um representando uma parcela, na qual se estudou o efeito dos regimes de umidade (com e sem estresse), enquanto as subparcelas corresponderam aos diferentes clones dentro de cada tanque.

\section{Resultados e Discussão}

\section{Altura da planta}

A análise da variância para a altura da planta mostrou que houve diferença significativa $(\mathrm{P}<0,05)$ para regimes de umidade, cultivares e a interação regimes de umidade x cultivares (Tabela 1 ). Pelos

Tabela 1 - Altura da planta de cultivares de capim-elefante e de um híbrido de capim-elefante $x$ milheto submetidos a diferentes regimes de umidade

Table 1 - Plant height of elephant grass cultivars and an elephant grass $x$ pearl millet hybrid submitted to different humidity regimes

\begin{tabular}{|c|c|c|}
\hline \multirow[t]{2}{*}{ Cultivar } & \multicolumn{2}{|c|}{$\begin{array}{c}\text { Altura da planta }(\mathrm{cm}) \\
\text { Plantheight } \\
\end{array}$} \\
\hline & $\begin{array}{c}\text { Sem estresse } \\
\text { hídrico } \\
\text { Withoutwater } \\
\text { stress }\end{array}$ & $\begin{array}{c}\text { Com estresse } \\
\text { hídrico } \\
\text { With water } \\
\text { stress }\end{array}$ \\
\hline Cameroon & $113,3^{\mathrm{Aab}}$ & $64,9^{\mathrm{Bb}}$ \\
\hline Roxo de Botucatu & $93,3^{\mathrm{Ac}}$ & $60,6^{\mathrm{Bb}}$ \\
\hline Mott & $99,9^{\mathrm{Abc}}$ & $72,5^{\mathrm{Bab}}$ \\
\hline Híbrido HV-241 & $120,2^{\mathrm{Aa}}$ & $85,9^{\mathrm{Ba}}$ \\
\hline
\end{tabular}

Valores na linha/coluna, seguidos de letras maiúsculas/minúsculas diferentes, são diferentes pelo teste Tukey $(P \leq 0,05)$.

$\mathrm{CV}(\%)$ : regimes de umidade $=4,26$; cultivares $=6,48$.

$\mathrm{F}$ : regime de umidade $\left({ }^{*}\right)$; cultivar $\left({ }^{*}\right)$; interação $\left({ }^{*}\right)$.

* Valores diferentes pelo teste $F(P \leq 0,05)$.

Values within a row/column, followed by capital/small letters, are different by

Tukey test $(P \leq .05)$.

CV $(\%)$ : humidity regimes $=4,26$; cultivars $=6.48$

F: humidity regimes $\left({ }^{*}\right)$; cultivar $\left({ }^{*}\right)$; interaction $\left({ }^{*}\right)$.

* Values are different by $F$ test $(P \leq .05)$. 
Rev. bras. zootec.

dados obtidos, observa-se que a altura das plantas foi severamente reduzida pelo estresse hídrico em todos os cultivares. Sob regime irrigado, o híbrido HV-241 e o cultivar Cameroon apresentaram as maiores alturas, diferindo estatisticamente do cultivar Roxo de Botucatu, que teve a menor altura. O cultivar Mott teve comportamento intermediário, não diferindo do Cameroon e do Roxo de Botucatu. Sob regime de estresse, o híbrido HV-241 também apresentou a maior altura, embora não diferiu estatisticamente do cultivar Mott. Todavia, o cultivar Cameroon, que sob regime irrigado se mostrou como um dos materiais mais altos, apresentou, juntamente com o Roxo de Botucatu, alturas significativamente $(\mathrm{P}<0,05)$ menores. Assim, observou-se que o cultivar Cameroon foi o que mais diminuiu de tamanho $(42,7 \%)$, quando submetido a estresse hídrico. Neste sentido, LIRA et al. (1989) afirmaram que muitas vezes os cultivares maiores e mais produtivos em condições de umidade adequada são os que mais sofrem em decorrência do estresse hídrico. Entretanto, este comportamento não foi verificado no híbrido HV-241, que apresentou a maior altura nos dois regimes de umidade, o que pode estar associado ao fato desse material ser mais precoce (SOUZA, 1971), aproveitando melhor a umidade inicial do solo, e também por apresentar uma maior resistência à seca, herdada do milheto (SINGH et al., 1983).

A redução do crescimento e, consequentemente, da altura da planta é apontada como um dos principais sintomas do estresse hídrico (RITCHIE, 1975). Tal comportamento também foi observado por AGUILAR CHAVARRIA (1985), em sete cultivares de capimelefante; por MACHADO et al. (1983), em Andropogon gayanus, Cenchrus ciliaris e Brachiaria humidicola; e por DIAS FILHO et al. (1989), em Panicum maximum.

Número de internódios por perfilho

A análise da variância mostrou diferença significativa $(\mathrm{P}<0,05)$ para regimes de umidade e cultivares, bem como para a interação regimes de umidade $\mathrm{x}$ cultivares (Tabela 2). O estresse hídrico afetou significativamente o número de internódios por perfilho, sendo que nenhum dos materiais submetidos a estresse apresentou internódios na altura em que foram cortados, demonstrando o efeito inibitório do estresse hídrico sobre o alongamento do caule. AGUILAR CHAVARRIA (1985) também verificou diminuição do número de internódios por perfilho, como consequência do estresse hídrico, passando de 8,0 unidades, nos tratamentos irrigados, para 6,62 nos
Tabela 2 - Número de internódios por perfilho de cultivares de capim-elefante e de um híbrido de capimelefante $x$ milheto submetidos a diferentes regimes de umidade

Table 2 - Number of internodes per tiller of elephant grass cultivars and an elephant grass $x$ pearl millet hybrid submitted to different humidity regimes

\begin{tabular}{lcc}
\hline Cultivar & $\begin{array}{c}\text { Número de internódios por perfilho } \\
\text { Number of internodes per tiller }\end{array}$ \\
\cline { 2 - 3 } & $\begin{array}{c}\text { Sem estresse } \\
\text { hídrico } \\
\text { Without water } \\
\text { stress }\end{array}$ & $\begin{array}{c}\text { Com estresse } \\
\text { hídrico } \\
\text { With water } \\
\text { stress }\end{array}$ \\
\hline Cameroon & $1,3^{\mathrm{Ac}}$ & $0^{\mathrm{Ba}}$ \\
Roxo de Botucatu & $1,1^{\mathrm{Ac}}$ & $0^{\mathrm{Ba}}$ \\
Mott & $2,1^{\mathrm{Ab}}$ & $0^{\mathrm{Ba}}$ \\
HíbridoHV-241 & $3,8^{\mathrm{Aa}}$ & $0^{\mathrm{Ba}}$ \\
\hline
\end{tabular}

Valores na linha/coluna, seguidos de letras maiúsculas/minúsculas diferentes, são diferentes pelo teste Tukey $(\mathrm{P} \leq 0,05)$.

CV $(\%)$ : regimes de umidade $=17,64$; cultivares $=4,75$.

F: regime de umidade $\left({ }^{*}\right)$; cultivar $\left({ }^{*}\right)$; interação $\left({ }^{*}\right)$.

* Valores diferentes pelo teste $\mathrm{F}(\mathrm{P} \leq 0,05)$.

Values within a row/column, followed by capital/small letters, are different by Tukey test $(P \leq .05)$

CV (\%): humidity regimes $=17.64 ;$ cultivars $=4.75$.

F: humidity regimes $\left({ }^{*}\right)$; cultivar $\left({ }^{*}\right)$; interaction $\left({ }^{*}\right)$.

* Values are different by $F$ test $(P \leq .05)$.

que sofreram estresse hídrico.

Entre os materiais irrigados, constatou-se que o híbrido HV-241 foi o que apresentou o maior número de internódios por perfilho, diferindo estatisticamente do cultivar Mott, que, por sua vez, foi significativamente superior aos cultivares Cameroon e Roxo de Botucatu, que apresentaram os menores números de internódios por perfilho.

\section{Comprimento e largura da lâmina foliar}

A análise da variância do comprimento da lâmina foliar mostrou diferença significativa apenas para os regimes de umidade (Tabela 3 ).

Independente do cultivar, o estresse hídrico promoveu redução de $28,47 \%$ no comprimento da lâmina foliar, passando de $69,9 \mathrm{~cm}$, nas parcelas irrigadas, para $50,0 \mathrm{~cm}$, nas que sofreram estresse hídrico. Essa redução está de acordo com a afirmação de LEA et al. (1992) de que a expansão foliar é particularmente sensível ao déficit hídrico, uma vez que as células só crescem quando estão túrgidas (FELIPPE, 1985).

Com relação à largura da lâmina foliar (Tabela 4), a análise da variância mostrou diferença significativa para os regimes de umidade e a interação regimes de umidade $\mathrm{x}$ cultivares. Não se verificou diferença significativa entre os cultivares sob regime de estresse hídrico. Sob regime irrigado, foi verificada diferença significativa apenas entre o cultivar Mott, que apre- 
Tabela 3 - Comprimento da lâmina foliar de cultivares de capim-elefante e de um híbrido de capim-elefante $x$ milheto submetidos a diferentes regimes de umidade

Table 3 - Leaf blade length of elephant grass cultivars and an elephant grass $x$ pearl millet hybrid submitted to different humidity regimes

\begin{tabular}{lccc}
\hline Cultivar & $\begin{array}{c}\text { Comprimento da lâmina foliar }(\mathrm{cm}) \\
\text { Leafblade lenght }\end{array}$ & \\
\cline { 2 - 3 } & $\begin{array}{c}\text { Sem estresse } \\
\text { hídrico } \\
\text { Without water } \\
\text { stress }\end{array}$ & $\begin{array}{c}\text { Com estresse } \\
\text { hídrico } \\
\text { With water } \\
\text { stress }\end{array}$ & \\
\hline Cameroon & 70,8 & 48,1 & $59,5^{\mathrm{a}}$ \\
Roxo de Botucatu & 70,2 & 44,6 & $57,4^{\mathrm{a}}$ \\
Mott & 70,5 & 47,6 & $59,0^{\mathrm{a}}$ \\
HíbridoHV-241 & 68,2 & 59,6 & $63,9^{\mathrm{a}}$ \\
$\overline{\mathrm{X}}$ & $69,9^{\mathrm{A}}$ & $50,0^{\mathrm{B}}$ & \\
\hline
\end{tabular}

Valores na linha/coluna, seguidos de letras maiúsculas/minúsculas diferentes, são diferentes pelo teste Tukey $(P \leq 0,05)$.

CV $(\%)$ : regimes de umidade $=6,93$; cultivares $=11,28$.

F: regime de umidade $\left(^{*}\right)$; cultivar (NS); interação (NS).

* Valores diferentes pelo teste $F(P \leq 0,05)$.

Values within a row/column, followed by capital/small letters, are different by Tukey test $(P \leq .05)$.

CV $(\%)$ : humidity regimes $=6.93$; cultivars $=11.28$.

F: humidity regimes $\left({ }^{*}\right)$; cultivar (NS); interaction (NS).

* Values are different by $F$ test $(P \leq .05)$.
Tabela 4 - Largura da lâmina foliar de cultivares de capimelefante e de um híbrido de capim-elefante $x$ milheto submetidos a diferentes regimes de umidade

Table 4 - Leaf blade width of elephant grass cultivars and an elephant grass $x$ pearl millet hybrid submitted to different humidity regimes

Cultivar Número de internódios por perfilho Number of internodes per tiller

\begin{tabular}{lcc}
\cline { 2 - 3 } & $\begin{array}{c}\text { Sem estresse } \\
\text { hídrico } \\
\text { Withoutwater } \\
\text { stress }\end{array}$ & $\begin{array}{c}\text { Com estresse } \\
\text { hídrico } \\
\text { With water } \\
\text { stress }\end{array}$ \\
\hline Cameroon & $1,8 \mathrm{~A}^{\mathrm{ab}}$ & $0,6^{\mathrm{Ba}}$ \\
Roxo de Botucatu & $1,7 \mathrm{~A}^{\mathrm{ab}}$ & $0,5^{\mathrm{Ba}}$ \\
Mott & $2,1^{\mathrm{Aa}}$ & $0,5^{\mathrm{Ba}}$ \\
HíbridoHV-241 & $1,6^{\mathrm{Ab}}$ & $0,7^{\mathrm{Ba}}$ \\
\hline
\end{tabular}

Valores na linha/coluna, seguidos de letras maiúsculas/minúsculas diferentes, são diferentes pelo teste Tukey $(P \leq 0,05)$.

CV $(\%)$ : regimes de umidade $=31,39$; cultivares $=15,93$.

F: regime de umidade $\left({ }^{*}\right)$; cultivar (NS); interação $\left({ }^{*}\right)$.

* Valores diferentes pelo teste $F(P \leq 0,05)$.

Values within a row/column, followed by capital/small letters, are different by Tukey test $(P \leq .05)$.

CV $(\%)$ : humidity regimes $=31.39$; cultivars $=15.93$.

F: humidity regimes $\left(^{*}\right)$; cultivar (NS); interaction $\left(^{*}\right)$.

* Values are different by $F$ test $(P \leq .05)$. sentou a maior largura da lâmina foliar $(2,1 \mathrm{~cm})$, e o híbrido HV-241, que apresentou as folhas mais estreitas $(1,6 \mathrm{~cm})$. Os cultivares Cameroon e Roxo de Botucatu tiveram folhas de largura intermediária, não diferindo dos demais.

A largura da lâmina foliar foi negativamente influenciada pelo estresse hídrico, sendo que, nas parcelas submetidas a estresse, os valores corresponderam a 23,8; 29,4; 33,3; e 43,7\% da largura das folhas das plantas irrigadas, para os cultivares Mott, Roxo de Botucatu e Cameroon e para o híbrido HV-241, respectivamente. Essa diferença, porém, deveu-se principalmente ao fato de as folhas se apresentarem enroladas, o que é comum entre as gramíneas em condições de estresse hídrico, como forma de diminuir a dessecação (LARCHER, 1986).

\section{Perfilhamento}

A análise da variância mostrou diferença significativa $(\mathrm{P}<0,05)$ apenas para cultivares, embora tenha sido verificada tendência de o estresse hídrico reduzir o perfilhamento basilar, sobretudo no híbrido HV-241 (Tabela 5).

Foi observado que o híbrido HV-241 apresentou o maior número de perfilhos basilares por planta
Tabela 5 - Número de perfilhos basilares por planta, de cultivares de capim-elefante e de um híbrido de capim-elefante $x$ milheto submetidos a diferentes regimes de umidade

Table 5 - Number of basal tillers per plant of elephant grass cultivars and an elephant grass $x$ pearl millet hybrid submitted to different humidity regimes

\begin{tabular}{|c|c|c|c|}
\hline \multirow[t]{3}{*}{ Cultivar } & \multirow{2}{*}{\multicolumn{2}{|c|}{$\begin{array}{l}\text { Número de perfilhos basilares } \\
\text { por planta } \\
\text { Number of basal tillers per plant }\end{array}$}} & \multirow[b]{3}{*}{$\bar{X}$} \\
\hline & & & \\
\hline & $\begin{array}{l}\text { Sem estresse } \\
\text { hídrico } \\
\text { Without water } \\
\text { stress } \\
\end{array}$ & $\begin{array}{l}\text { Com estresse } \\
\text { hídrico } \\
\text { With water } \\
\text { stress } \\
\end{array}$ & \\
\hline Cameroon & 70,8 & 48,1 & $59,5^{\mathrm{a}}$ \\
\hline Cameroon & 1,7 & 1,7 & $1,7^{\mathrm{b}}$ \\
\hline Roxo de Botucatu & 2,1 & 1,9 & $2,0^{\mathrm{b}}$ \\
\hline Mott & 1,7 & 1,6 & $1,6^{\mathrm{b}}$ \\
\hline Híbrido HV-241 & 7,1 & 3,7 & $5,4 a$ \\
\hline$\overline{\mathrm{X}}$ & $3,1^{\mathrm{A}}$ & $2,2^{\mathrm{A}}$ & \\
\hline \multicolumn{4}{|c|}{$\begin{array}{l}\text { Valores na linha/coluna, seguidos de letras maiúsculas/minúsculas } \\
\text { diferentes, são diferentes pelo teste Tukey }(P \leq 0,05) . \\
\text { CV }(\%) \text { : regimes de umidade }=18,47 ; \text { cultivares }=15,05 . \\
\text { F: regime de umidade }(\mathrm{NS}) ; \text { cultivar }\left({ }^{*}\right) ; \text { interação }(\mathrm{NS}) . \\
\text { * Valores diferentes pelo teste } \mathrm{F}(\mathrm{P} \leq 0,05) \text {. } \\
\text { Values within a row/column, followed by capital/small letters, are different by } \\
\text { Tukey test }(P \leq .05) \text {. } \\
\text { CV }(\%) \text { : humidity regimes }=18.47 \text {; cultivars }=15.05 . \\
\left.\text { F: humidity regimes }(N S) ; \text { cultivar }{ }^{*}\right) \text {; interaction }(N S) . \\
\text { * Values are different by } F \text { test }(P \leq .05) \text {. }\end{array}$} \\
\hline
\end{tabular}


$(5,4)$, diferindo dos cultivares Roxo de Botucatu, Cameroon e Mott, que apresentaram números semelhantes $(2,0 ; 1,7$; e 1,6 , respectivamente). Todavia, SANTOS (1996) não observou diferença significativa entre o híbrido HV-241 e os cultivares Australiano e Cameroon, quanto ao número de perfilhos basilares, embora o híbrido tenha apresentando maior número do que os cultivares.

Para o número de perfilhos axilares, conforme os dados apresentados na Tabela 6 , a análise da variância mostrou diferença significativa para cultivares e para a interação regimes de umidade $\mathrm{x}$ cultivares. Apenas o híbrido HV-241 teve o número de perfilhos axilares significativamente reduzido pelo estresse hídrico, passando de 4,3 perfilhos por planta, no regime irrigado, para 2,1 perfilhos, em condições de estresse. Em condições irrigadas, o híbrido HV-241 apresentou o maior número de perfilhos axilares $(4,3)$; o cultivar Roxo de Botucatu, número intermediário $(2,2)$; e o Mott e o Cameroon, números semelhantemente inferiores $(0,9$ e 0,8$)$. Entretanto, os materiais não diferiram entre si sob o regime de estresse hídrico.

BOTREL et al. (1991) observaram tendência de a irrigação aumentar o número de perfilhos basais, porém esta não teve efeito sobre o perfilhamento aéreo de sete cultivares de capim-elefante.

Com relação ao número total de perfilhos por

Tabela 6 - Número de perfilhos axilares por planta, de cultivares de capim-elefante e de um híbrido de capim-elefante $x$ milheto submetidos a diferentes regimes de umidade

Table 6 - Number of lateral tillers per plant of elephant grass cultivars and an elephant grass $x$ pearl millet hybrid submitted to different humidity regimes

\begin{tabular}{lcc}
\hline Cultivar & $\begin{array}{c}\text { Número de perfilhos axilares por planta } \\
\text { Number of lateral tillers per plant }\end{array}$ \\
\cline { 2 - 3 } & $\begin{array}{c}\text { Sem estresse } \\
\text { hídrico } \\
\text { Without water } \\
\text { stress }\end{array}$ & $\begin{array}{c}\text { Com estresse } \\
\text { hídrico } \\
\text { With water } \\
\text { stress }\end{array}$ \\
\hline Cameroon & $0,8^{\mathrm{Ac}}$ & $1,6^{\mathrm{Aa}}$ \\
Roxo de Botucatu & $2,2^{\mathrm{Ab}}$ & $1,8^{\mathrm{Aa}}$ \\
Mott & $0,9^{\mathrm{Ac}}$ & $1,1^{\mathrm{Aa}}$ \\
HíbridoHV-241 & $4,3^{\mathrm{Aa}}$ & $2,1^{\mathrm{Ba}}$ \\
\hline
\end{tabular}

Valores na linha/coluna, seguidos de letras maiúsculas/minúsculas diferentes, são diferentes pelo teste Tukey $(\mathrm{P} \leq 0,05)$.

CV $(\%)$ : regimes de umidade $=6,93$; cultivares $=13,08$.

F: regime de umidade (NS); cultivar $\left({ }^{*}\right)$; interação $\left({ }^{*}\right)$.

* Valores diferentes pelo teste $F(P \leq 0,05)$.

Values within a row/column, followed by capital/small letters, are different by

Tukey test $(P \leq .05)$.

$C V(\%)$ : humidity regimes $=6.93 ;$ cultivars $=13.08$.

F: humidity regimes (NS); cultivar $\left(^{*}\right)$; interaction $\left(^{*}\right)$.

* Values are different by $F$ test $(P \leq .05)$. planta, pode-se observar na Tabela 7 que houve diferença significativa para cultivares e para a interação regimes de umidade $\mathrm{x}$ cultivares. Com exceção do híbrido HV-241, o estresse hídrico não reduziu o número total de perfilhos dos cultivares. Em condições irrigadas, o híbrido HV-241 destacou-se como o material que mais perfilhou, com média de 11,4 perfilhos/ planta, enquanto os cultivares Roxo de Botucatu, Mott e Cameroon não diferiram entre si, com médias de 4,$3 ; 2,6$; e 2,5 perfilhos/ planta, respectivamente.

Sob regime de estresse hídrico, apesar de o híbrido HV-241 ter o número total de perfilhos diminuído, continuou sendo o material que mais perfilhou (5,8 perfilhos/planta), embora não tenha diferido significativamente do cultivar Roxo de Botucatu, que apresentou 3,7 perfilhos/planta. Os cultivares Cameroon e Mott, com 3,3 e 2,7 perfilhos/planta, foram significativamente inferiores ao híbrido, porém semelhantes ao Roxo de Botucatu. O maior perfilhamento observado no híbrido HV-241 está de acordo com o relato de BOGDAN (1977), o qual afirmou que os híbridos de capim-elefante com o milheto apresentam maior número de perfilhos em relação ao capim-elefante.

Há certa controvérsia quanto à influência do estresse hídrico sobre o perfilhamento de gramíneas,

Tabela 7 - Número total de perfilhos por planta, de cultivares de capim-elefante e de um híbrido de capimelefante $x$ milheto submetidos a diferentes regimes de umidade

Table 7 - Total number of tillers per plant of elephant grass cultivars and an elephant grass $x$ pearl millet hybrid submitted to different humidity regimes

\begin{tabular}{lcc}
\hline Cultivar & $\begin{array}{c}\text { Número de perfilhos axilares por planta } \\
\text { Number of lateral tillers per plant }\end{array}$ \\
\cline { 2 - 3 } & $\begin{array}{c}\text { Sem estresse } \\
\text { hídrico } \\
\text { Without water }\end{array}$ & $\begin{array}{c}\text { Com estresse } \\
\text { hídrico } \\
\text { stress }\end{array}$ \\
& $2,5^{\mathrm{Ab}}$ & With water \\
Cameroon & $4,3^{\mathrm{Ab}}$ & $3,3^{\mathrm{Ab}}$ \\
Roxo de Botucatu & $2,6^{\mathrm{Ab}}$ & $3,7^{\mathrm{Aab}}$ \\
Mott & $11,4^{\mathrm{Aa}}$ & $2,7^{\mathrm{Ab}}$ \\
HíbridoHV-241 & $5,8^{\mathrm{Ba}}$ \\
\hline
\end{tabular}

Valores na linha/coluna, seguidos de letras maiúsculas/minúsculas diferentes, são diferentes pelo teste Tukey $(P \leq 0,05)$.

CV $(\%)$ : regimes de umidade $=13,07$; cultivares $=10,63$.

F: regime de umidade (NS); cultivar $\left({ }^{*}\right)$; interação $\left({ }^{*}\right)$.

* Valores diferentes pelo teste $\mathrm{F}(\mathrm{P} \leq 0,05)$.

Values within a row/column, followed by capital/small letters, are different by

Tukey test $(P \leq .05)$.

CV (\%): humidity regimes $=13.07$; cultivars $=10.63$.

$F$ : humidity regimes (NS); cultivar $\left({ }^{*}\right)$; interaction $\left({ }^{*}\right)$.

* Values are different by $F$ test $(P \leq .05)$. 
sendo que alguns autores afirmam que o perfilhamento é inibido pelo déficit hídrico (LAUDE, 1972; DIAS FILHO et al., 1989; BUXTON e FALES, 1994), enquanto outros não observaram tal influência (AGUILAR CHAVARRIA, 1985). Entretanto, tal fato pode estar associado com a fase do ciclo da planta em que ocorre o estresse.

\section{Conclusões}

Com exceção do perfilhamento, o estresse hídrico promoveu redução nos demais parâmetros morfológicos estudados.

O efeito do estresse hídrico foi maior que o dos cultivares, para as variáveis altura da planta, número de internódios por perfilho e comprimento e largura da lâmina foliar.

O híbrido HV-241 apresentou-se igual ou superior aos cultivares em todas as variáveis estudadas, exceto a largura da folha, que foi maior no cv. Mott, na ausência de estresse hídrico

\section{Referências Bibliográficas}

AGUILAR CHAVARRIA, J.A. Avaliação da sobrevivência ao estresse hídrico e de outras características morfofisiológicas de sete clones de capim-elefante (Pennisetum purpureum Schum.) em condições controladas. Recife: UFRPE, 1985. 189p. Dissertação (Mestrado em Produção Animal) Universidade Federal Rural de Pernambuco, 1985.

BEGG, J. E. 1980. Morphological adaptations of leaves to water stress. In: TURNER, N.C., KRAMER, P.J. (Eds.) Adaptation of plants to water and high temperature stress. New York: John Wiley and Sons. p.33-42.

BOGDAN, A.V. 1977. The grasses. In: BOGDAN, A.V. (Ed.) Tropical pasture and fodder plants (grasses and legumes). London: Longman. p.1-301.

BOTREL, M.A., ALVIM, M.J., XAVIER, D.F. 1991. Efeito da irrigação sobre algumas características agronômicas de cultivares de capim-elefante. Pesq. Agropec. Bras., 26(10):1731-1736.

BUXTON, D.R., FALES, S.L. 1994. Plant environment and quality. In: FAHEY JR., G.C. (Ed.) Forage quality, evaluation and utilization. Madison: American Society of Agronomy. p.155-199.

DIAS FILHO, M.B., CORSI, M., CUSATO, S. 1989. Respostas morfológicas de Panicum maximum Jacq. cv. Tobiatã ao estresse hídrico. Pesq. Agropec. Bras., 24(7):893-898.

FELIPPE, G.M. 1985. Desenvolvimento. In: FERRI, M.G. (Ed.) Fisiologia vegetal. 2.ed. São Paulo: EPU. v.2, p.1-37.
HSIAO, T.C., ACEVEDO, E. 1975. Plant responses to water deficits, water-use efficiency, and drought resistence. In: STONE, J. F. (Ed.)Plant modification for more efficient water use. Amsterdam: Elsevier Scientific Publishing Company. p.59-84.

KRAMER, P.J. 1983. Water relations of plants. New York: Academic Press. 489p.

LARCHER, W. 1986. Ecofisiologia vegetal. São Paulo: EPU. 319 p.

LAUDE, H.M. 1972. External factors affecting tiller development. In: YOUNGNER, V.B., McKELL, C.M. (Eds.) The biology and utilization of grasses. New York: Academic Press. p.146-154.

LEA, P.J., AL-SUlAITI, A., PALMER, S. et al. Absorção e metabolismo de nitrogênio sob estresse hídrico. In: SIMPÓSIO INTERNACIONAL SOBRE ESTRESSE AMBIENTAL: O MILHO EM PERSPECTIVA, 1992, Belo Horizonte. Resumos... Belo Horizonte: EMBRAPA-CNPMS, 1992. p.26-27.

LIRA, M.A., BRANDÃO, A.R.M., TABOSA, J.N. et al. 1989. Estudos de resistência à seca em genótipos de sorgo forrageiro (Sorghum bicolor (L.) Moench). R. Soc. Bras. Zootec., 18(1):1-12.

MACHADO, R.C.R., SOUZA, H.M.F., MORENO, M.A. et al. 1983. Variáveis relacionadas com a tolerância de gramíneas forrageiras ao déficit hídrico. Pesq. Agropec. Bras., 18(6):603-608.

MEIDNER, H., SHERIFF, D.W. 1976. The role of water in plants. In: MEIDNER, H., SHERIFF, D.W. (Eds.) Water and Plants. New York: John Wiley \& Sons. p.128-135.

RITCHIE, J. T. 1975. Atmospheric and soil water influences on the plant water balance. In: STONE, J.F. (Ed.) Plant modification for more efficient water use. Amsterdam: Elsevier Scientific Publishing Company. p.183-198.

SANTOS, M.C.S. Avaliação de 4 clones de Pennisetum sob diferentes níveis de estresse hídrico. Recife: UFRPE, 1996. 117p. Dissertação (Mestrado em Botânica) - Universidade Federal Rural de Pernambuco, 1996.

SINGH, P., KANEMASU, E.T., SINGH, P. 1983. Yield and water relations of pearl millet genotypes under irrigated and non irrigated conditions. Agron. J., 75(6):886-890.

SOUZA, R.M. Avaliação de híbridos de capim-elefante (Pennisetum purpureum Schumacher) com pearl millet (Pennisetum typhoides (Burm.) Stapf e Hubbard) e seus progenitores. Viçosa: UFV, 1971.38p. Dissertação (Mestrado em Zootecnia) - Universidade Federal de Viçosa, 1971.

SUÁREZ, J.J., HERRERA, J., HERRERA, R.S. 1986. Riego. In: SISTACHS, M. (Ed.) Los Pastos en Cuba - Producción. 2.ed. Havana: Edica, v.1, p.417-468.

Recebido em: 17/04/00

Aceito em: 14/08/00 